\title{
Identification of key factors determining the surface oil concentration of encapsulated lipid particles produced by spray drying
}

\author{
Linke, A. ${ }^{a^{*}}$; Balke, T. ${ }^{\text {a }}$; Kohlus, R. ${ }^{\text {a }}$
}

a Department of Food Powders and Process Engineering. University of Hohenheim, Stuttgart, Germany

*E-mail of the corresponding author: annika.linke@uni-hohenheim.de

\begin{abstract}
Potential factors leading to surface oil were investigated by analyzing the impact of emulsion properties, atomization and drying conditions separately. An increased oil load, droplet size and in particular the size of droplet aggregates led to significant more surface oil. Increasing the viscosity, inlet temperature and relative humidity resulted in larger particles with a higher encapsulation efficiency. The results indicate that the probability of oil droplets being in contact with the particle surface determines the amount of surface oil. Oil diffusion towards the surface was excluded due to the short residence times and high viscosities.
\end{abstract}

Keywords: microencapsulation; encapsulation efficiency; emulsion properties; atomization; drying conditions 


\section{Introduction}

Polyunsaturated fatty acids are susceptible to oxidation. Microencapsulation is a strategy to protect lipids against environmental oxygen by embedding oil droplets in a solid matrix acting as an oxygen diffusion barrier. Some droplets are not fully covered and are in contact with the particle surface. This so-called surface oil is not protected and directly exposed to environmental oxygen.

In literature several hypotheses about surface oil creation during spray drying are stated. Apart from emulsion stability, oil droplet disruption during atomization and oil diffusion towards the particle surface are believed to be the main factors leading to surface oil [1]-[3]. Experimental confirmation is partly lacking due to the high number of potential factors interacting with each other and influencing the encapsulation efficiency (EE).

In this study it is hypothesized that the probability of oil droplets being in contact with the particle surface determines the surface oil concentration. The aim of this study is to identify key factors determining the EE by investigating the impact of emulsion properties, feed atomization and drying rate separately.

\section{Materials and Methods}

High quality fish oil (Omega Oil 1812 TG Gold) was kindly provided by BASF Personal Care and Nutrition GmbH (Illertissen, Germany). Rapeseed oil, maltodextrin DE21 (AGENABON) and Soy Protein Isolate (Vegacon 90) were obtained from Herny Lamotte Oils GmbH (Bremen, Germany), Agrana GmbH (Frankfurt am Main, Germany) and Eurosoy GmbH (Hamburg, Germany), respectively.

\subsection{Emulsion and Powder Preparation}

Soy protein and maltodextrin DE21 were hydrated in distilled water for $12 \mathrm{~h}$. Oil was dispersed by operating an IKA SPP25 (Staufen, Germany) for $10 \mathrm{~min}$. The emulsion was homogenized in four passes using the homogenizer HL 1.3-400KX (HST Maschinenbau $\mathrm{GmbH}$, Dassow, Germany). Emulsions were spray dried using a pilot plant dryer (type FSD 4.0, GEA-Niro, Copenhagen, Denmark) with a maximum water evaporation capacity of 30 $\mathrm{kg} / \mathrm{h}$ operated at an airflow of $200 \mathrm{~kg} / \mathrm{h}$ in the closed loop mode. The feed was atomized using a two fluid nozzle set to 1 bar (ex GEA-Niro). Inlet and outlet temperature were set to $180^{\circ} \mathrm{C}$ and $85^{\circ} \mathrm{C}$, respectively.

\subsection{Analysis}

\subsubsection{Oil droplet size}

Static light scattering: A Mastersizer 2000 (Malvern Instruments Ltd, UK) was used to analyze the oil droplet size according to Mie-Theory. Three samples of each emulsion were measured in triplicate. 
Pulsed field gradient: A Bruker minispec mq 20 NMR Analyzer (Bruker, Rheinstetten, Germany), operating at $20 \mathrm{MHz}$ and equipped with a controlled pulsed gradient unit with a probe head H20-10-25-AVXG (Bruker, Rheinstetten, Germany) was operated at $20{ }^{\circ} \mathrm{C}$. The oil droplet size of emulsions and within the powder was determined according to the procedure described by Linke et al. (2017) [4].

\subsubsection{Particle Size Distribution}

A Mastersizer 2000 (Malvern Instruments Ltd, UK) was used to determine the particle size by dispersing the powder in ethanol. Data were analyzed according to Fraunhofer theory. Three samples of each powder were measured six times.

\subsubsection{Oil Load, Surface Oil and Encapsulation Efficiency}

The surface oil was removed applying a procedure described by Bae and Lee (2008) with some modifications [5]. Approximately $1 \mathrm{~g}$ powder and $10 \mathrm{~mL}$-Hexane (ROTISOLV® HPLC) were shaken for 2 min. The powder was separated by filtration and washed three times with $10 \mathrm{~mL}$ n-Hexane. The oil load of the untreated and washed powder was determined according to a method described by Linke et al (2017). Briefly, a Bruker Minispec MQ20 NMR Analyzer (Bruker, Rheinstetten, Germany) with an absolute probe head H2018-25-A1 was operated at $40^{\circ} \mathrm{C}$ with a resonance frequency of $19.95 \mathrm{MHz}$. The powder was weighed into tubes and tempered at $40^{\circ} \mathrm{C}$ for $45 \mathrm{~min}$. The instrument was calibrated with standards consisting of 0.07 and $0.9 \mathrm{~g}$ fish oil. The surface oil was calculated by subtracting the remaining oil from the total oil. The encapsulation efficiency was determined by dividing the amount of encapsulated oil by the total oil times 100 .

\section{Results}

\subsection{Impact of emulsion properties on the encpasualtion efficiency}

In order to vary the emulsion properties in terms of viscosity, oil content and oil droplet size the homogenization pressure, oil load, dextrose equivalent (DE) of the maltodextrin and the soy protein concentration were modified (Figure 1). With increasing ratio of soy protein to oil the $\mathrm{d}_{3,2}$ of oil droplets decreased (Figure 1A). Additionally, the diameter measured by static light scattering (SLS) and nuclear magnetic resonance (NMR) differed at concentrations below $0.07 \mathrm{~g}(\mathrm{w} / \mathrm{w})$ protein per oil. Due to different measurement principles, the latter method measures the size of individual droplets, whereas SLS determines the average diameter of droplet clusters [6], [7]. Insufficient concentrations of soy protein lead to droplet flocculation, which was confirmed by light microscopy (data not shown). Using maltodextrin with a higher DE led to a significant reduction of viscosity, due to shorter glucose chains (Figure 1B). Homogenizing emulsions at higher pressure decreased the oil droplet size, whereas the viscosity remained constant (Figure 1C). In case of an higher oil content the droplet size and viscosity slightly increased (Figure $1 \mathrm{D}$ ). 
Identification of key factors determining the surface oil concentration of encapsulated lipid particles produced by spray drying

$$
\square \mathrm{d}_{3,2} \mathrm{SLS} \bullet \mathrm{d}_{3,2} \mathrm{NMR} \boldsymbol{\Delta} \text { Viscosity }
$$

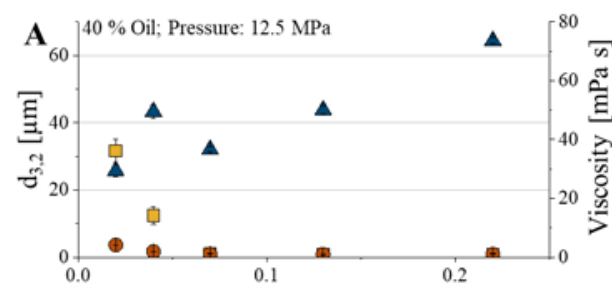

Soy protein per oil $(w / w)$

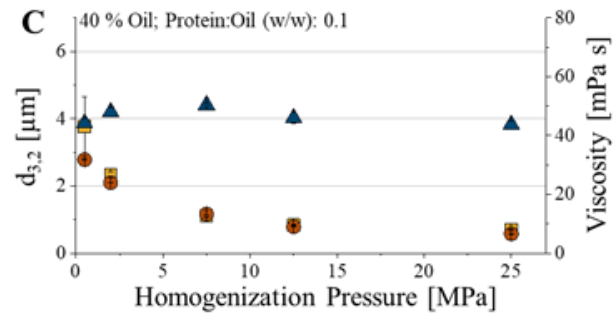

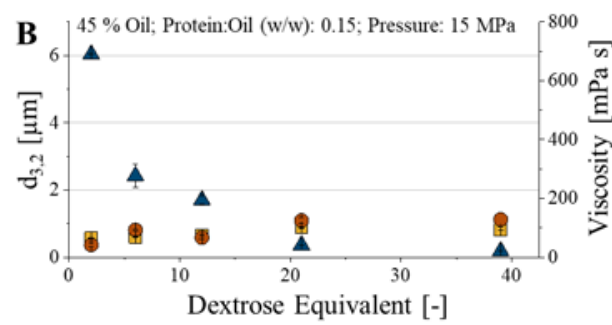

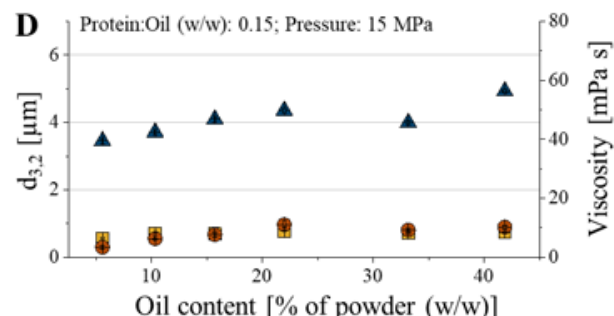

Figure 1: Emulsion viscosity and Sauter mean diameter $d_{3,2}$ of oil droplets determined by static light scattering (SLS) and nuclear magnetic resonance (NMR) as a function of the soy protein concentration (A), dextrose equivalent of maltodextrin (B) homogenization pressure (C) and oil content (D); default settings were set to a solid content of $45 \%$ and maltodextrin DE21

\subsubsection{Impact of the droplet size on the EE}

Modifying the emulsifier concentrations and homogenization pressure resulted in a change in the Sauter mean diameter of oil droplets (Figure 1). Plotting the $\mathrm{d}_{3,2}$ as a function of the EE shows that larger droplets lead to a less efficient encapsulation (Figure 2).
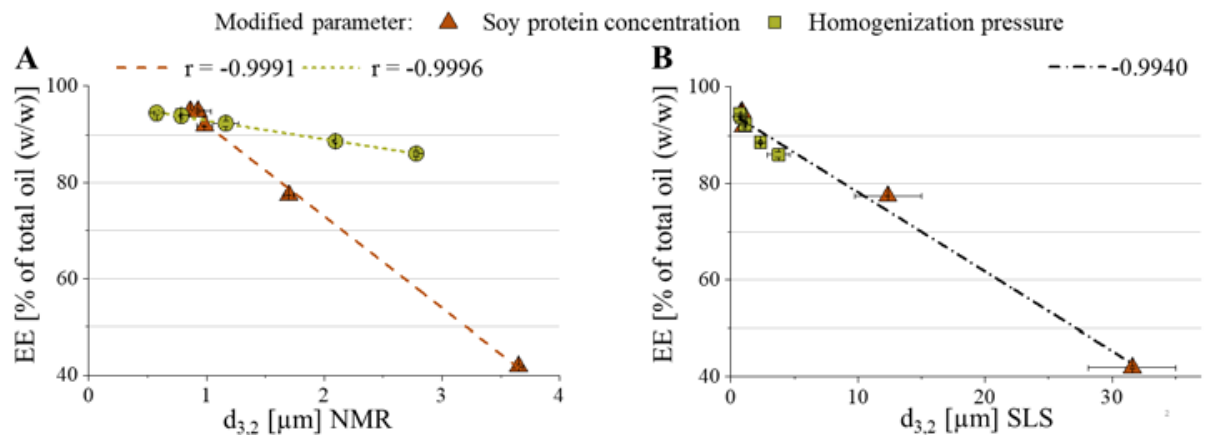

Figure 2: EE [\% of total oil] as a function of the Sauter mean diameter $d_{3,2}$ of oil droplets [ $\mu \mathrm{m}$ ], determined by nuclear magnetic resonance (NMR) (A) and static light scattering (SLS) (B)

In case of the $d_{3,2}$ determined by NMR, the droplet size modified by the homogenization pressure affects the EE less compared to the protein concentration (A), whereas the impact of the diameter measured by SLS is aligned for both samples sets (B). As SLS measures the 
size of droplet clusters it is concluded that apart from the individual size, oil aggregates lead to more surface oil. As droplet clusters are larger compared to individual droplets, it is more likely for them being in contact with the powder particle surface and contributing to surface oil.

\subsubsection{Impact of the oil load on the EE}

The EE and the amount of surface oil is plotted as a function of the oil concentration in the emulsion (Figure 3, A). A higher oil load leads to more surface oil supporting the hypothesis, as the likelihood for droplets being in contact with the particle surface is higher if more oil is present. However, the EE increases up to oil loads of $15 \%$ and decreases at higher concentrations. Even though less oil is located at the particle surface, the ratio to the amount of encapsulated oil fraction is smaller leading to a decreased encapsulation efficiency.
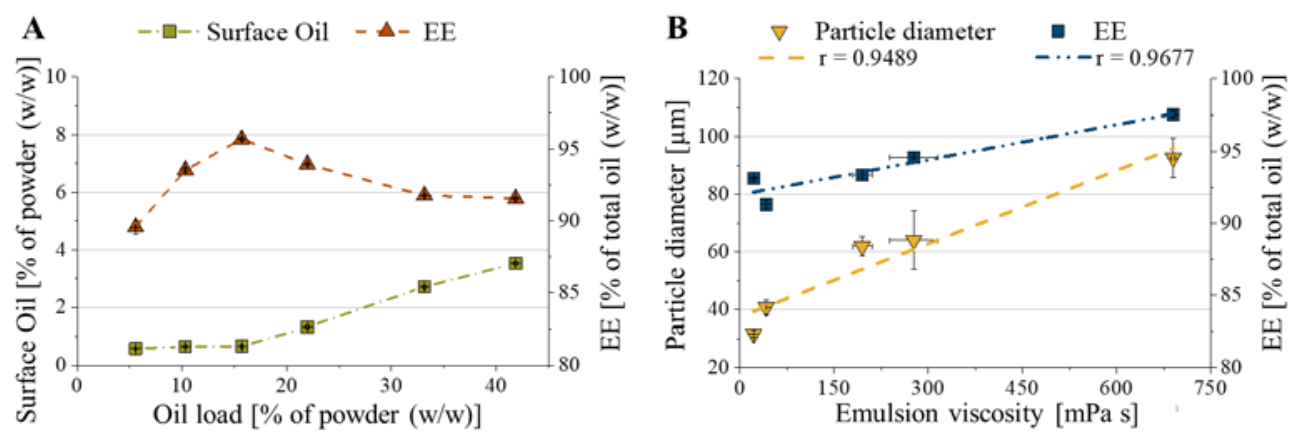

Figure 3: EE [\% of total oil] and surface oil as a function of the oil concentration in the emulsion (A); $E E$ and particle diameter $\mathrm{d}_{50,3}$ as a function of the viscosity for samples containing maltodextrin with a different dextrose equivalent (B)

\subsubsection{Impact of the viscosity}

Modifying the dextrose equivalent of the maltodextrin resulted in a change in the emulsion viscosity (Figure 1). With increasing viscosity larger particles and a more efficient encapsulation is obtained (Figure 3, B). It is assumed, that larger particles increase the EE due to a lower ratio of particle surface to particle volume. Thus, the viscosity is affecting the EE indirectly by changing the size of encapsulated lipid particles.

\subsection{Impact of drying conditions and particle properties}

Figure 4 shows the particle diameter and the EE as a function of the solid content, inlet temperature and relative humidity of the drying medium. A higher solid content and inlet temperature resulted in larger particles, due to a higher viscosity and faster crust formation preventing shrinkage, respectively. A higher humidity in the drying air resulted in slightly 
larger particles. This is unexpected, as the drying rate is supposed to decelerate enabling particle shrinkage.

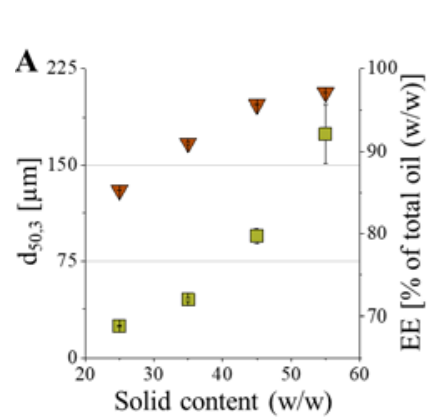

$\square$ Particle diameter $\mathrm{d}_{50,3} \nabla$ EE

Figure 4: Particle diameter $\mathrm{d}_{50,3}$ [ $\mathrm{\mu m}$ ] and EE [\% of total oil] as a function of the solid content, inlet temperature and relative humidity for samples differing in oil concentration and solid content, the default settings were set to a solid content and oil load of $35 \%$ and $30 \%$, respectively.

Plotting the EE as a function of the particle diameter shows an increase until a plateau is reached (Figure 5). Apart from the modified drying conditions, particles containing different dextrose equivalent are following the same trend. Larger particles have a lower particle surface to volume ratio. Thus, it is more likely for oil droplets being embedded in the particle center and not being in contact with the particle surface. Particles above approximately 100 $\mu \mathrm{m}$ are powder aggregates. The EE might reach a plateau as aggergates consist of a number of individual particles, which determine the particle surface. Hence, the surface is not decreasing further with increasing aggregate size.

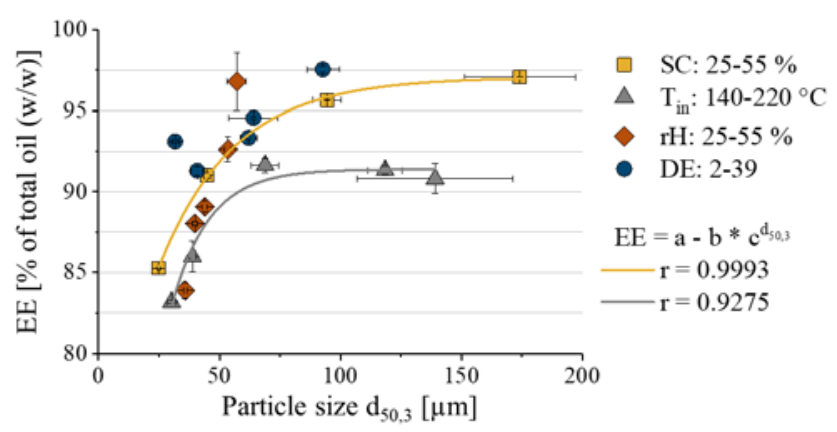

Figure 5: EE [\% of total oil] as a function of the particle diameter $d_{50,3}$ [ $[\mu \mathrm{m}]$; Samples contain different solid content (SC) and maltodextrin dextrose equivalent (DE) and are spray dried at different inlet temperatures $\left(\mathrm{T}_{\text {in }}\right)$ and relative humidity $(\mathrm{rH})$. 


\section{Discussion}

In this study the impact of potential factors on the encapsulation efficiency during the production of encapsulated lipid particles was investigated. In order to do so, the influence of emulsion properties and spray drying conditions on the surface oil creation was analyzed separately. Due to the number of potential factors and their interaction with each other it is challenging to identify the main contributors causing surface oil.

Smaller particles, oil droplets and in particular oil droplet clusters decreased the encapsulation efficiency significantly. This supports the hypothesis, that the probability of oil droplets being in contact with the particle surface determines the amount of surface oil. This might explain that encapsulated lipid particles produced by a benchtop spray dryer generally have a lower encapsulation efficiency compared to powders obtained on pilot plant scale as the obtained particles are smaller.

Larger particles were obtained at a higher solid content and inlet temperatures. Due to the increased viscosity and the fast drying the mobility of oil droplets was reduced, so that the likelihood of oil diffusing towards the particle surface is decreased. In terms of the increased humidity, the drying medium is more polar, which might prevent oil diffusion additionally. However, due to the short residence times and high viscosity it is suggested that oil droplet diffusion has no significant impact on the encapsulation efficiency.

Increasing the oil load up to $15 \%$ resulted in a higher efficiency even though the amount of surface oil was raising. Maximizing the concentration of lipids being fully embedded in the matrix increases the efficiency, but does not necessarily enhance the shelf life, as the absolute amount of surface oil being exposed to environmental oxygen may also increase. Additionally, to the investigated properties the volume of vacuoles in the particles should be considered, as it decreases the ratio of outer particle surface to the effective matrix volume. This might reduce the encapsulation efficiency, due to a higher likelihood of being in contact with the particle surface.

\section{Conclusions}

In this study the impact of the emulsion properties and spray drying conditions on the encapsulation efficiency was investigated. An increased oil load, smaller particles and larger oil droplets and droplet aggregates led to more surface oil. It supports the hypothesis, that the probability of oil droplets being in contact with the particle surface determines the success of the encapsulation. 


\section{Nomenclature}

$\begin{array}{llc}\mathrm{d}_{3,2} & \text { Sauter mean diameter } & \mu \mathrm{m} \\ \mathrm{EE} & \text { Encapsualtion Efficiency } & \% \\ \mathrm{DE} & \text { Dextrose Equivalent } & -\end{array}$

\section{Acknowledgements}

This work was supported by a grant for Annika Linke from the Ministry of Science, Research and the Arts of Baden-Württemberg (MWK) Az: 7533-10-5-87. Furthermore, the author acknowledges generous support by the bioeconomy graduate program BBW ForWerts, supported by the MWK

\section{References}

[1] S. M. Jafari, E. Assadpoor, Y. He, and B. Bhandari, "Encapsulation efficiency of food flavours and oils during spray drying,” Dry. Technol., vol. 26, no. 7, pp. 816-835, 2008.

[2] Y. Minemoto, K. Hakamata, S. Adachi, and R. Matsuno, "Oxidation of linoleic acid encapsulated with gum arabic or maltodextrin by spray-drying,” J. Microencapsul., vol. 19, no. 2, pp. 181-189, 2002.

[3] R. V. Tonon, C. R. F. Grosso, and M. D. Hubinger, "Influence of emulsion composition and inlet air temperature on the microencapsulation of flaxseed oil by spray drying," Food Res. Int., vol. 44, no. 1, pp. 282-289, 2011.

[4] A. Linke, T. Anzmann, J. Weiss, and R. Kohlus, “Advanced characterisation of encapsulated lipid powders regarding microstructure by time domain-nuclear magnetic resonance,” J. Microencapsul., vol. 34, no. 2, pp. 140-150, 2017.

[5] E. K. Bae and S. J. Lee, "Microencapsulation of avocado oil by spray drying using whey protein and maltodextrin,” J. Microencapsul., vol. 25, no. 8, pp. 549-560, 2008.

[6] G. J. W. Goudappel, J. P. M. Van Duynhoven, and M. M. W. Mooren, "Measurement of oil droplet size distributions in food oil/water emulsions by time domain pulsed field gradient NMR,” J. Colloid Interface Sci., vol. 239, no. 2, pp. 535-542, 2001.

[7] H. Todt, W. Burk, G. Guthausen, A. Guthausen, A. Kamlowski, and D. Schmalbein, “Quality control with time-domain NMR,” Eur. J. Lipid Sci. Technol., vol. 103, no. 12, pp. 835-840, 2001. 